\title{
A PROCEDURE FOR TUBE COUNT DETERMINATION IN SINGLE AND MULTIPLE PASS TUBULAR HEAT EXCHANGERS
}

\author{
M. S. Medeiros, ${ }^{\mathrm{a}}$ \\ and A. J. K. Leiroz ${ }^{b}$ \\ Universidade Federal do Rio de Janeiro \\ Departamento de Engenharia Mecânica \\ POLI/COPPE \\ P.O. Box. 68503 \\ Rio de Janeiro, RJ, Brazil \\ amichel@hvconsult.com.br \\ bleiroz@ufrj.br

\begin{abstract}
The development of a simple computational procedure that allows the precise determination of important parameters for the thermal and mechanical design of tubular heat exchangers is discussed in the present work. The design of tubular heat exchangers for a wide variety of applications can involve the use of empirical expressions and data tables for the determination of the tube bundle parameters, such as the tube count and the tube bundle outside diameter. The motivation for developing the discussed procedure resides in addressing cases for which empirical expressions are inapplicable or data table are unavailable. Initially, the shell positions in which tubes can be placed are determined based on specified tube pitch, angle of arrangement, inlet and outlet nozzle diameters and tube bundle-to-shell clearance. The maximum number of tubes for the given configuration is obtained from the tube position searching procedure. A sorting algorithm, based on the tube distance to the shell center, is used to appropriately place a specified number of tubes within the heat exchanger cross section. Results for a single- and multiple-pass fixedtubesheet heat exchangers are presented and compared with available tube count tables.

Keywords: Heat exchangers, tube count, industrial equipments, mechanical design, pressure vessels.
\end{abstract}

\section{NOMENCLATURE}

$a, c \quad$ angular coefficients of the searching lines

$b, d \quad$ linear coefficients of the searching lines

$D_{S} \quad$ heat exchanger shell inside diameter

$D$ tube outside diameter

$k$ tube order

$l_{\max }$

$l_{\text {min }}$

$t_{c}$ coefficient

minimum value of the straight-line linear coefficient

pass partition plate thickness

impingement plate thickness

horizontal position

vertical position

position of the first row of tube

position of the last row of tubes maximum value of the straight-line linear

\section{Greek symbols}

$\alpha \quad$ angle of the arrangement

$\Delta l \quad$ linear coefficient increment

$\Delta_{k} \quad$ tube-center to shell-center distance

$\varepsilon_{r} \quad$ tube bundle to shell radial clearance

$\varepsilon_{t p} \quad$ impingement plate to tube walls clearance

$\theta \quad$ complementary angle of $\alpha$

$\sigma \quad$ tube pitch

$\phi_{\text {in }} \quad$ inlet nozzle internal diameter

$\phi_{\text {out }} \quad$ outlet nozzle internal diameter

\section{INTRODUCTION}

Shell-and-tube heat exchangers are process equipment within which energy is transferred between two convective fluid streams at different temperatures through a solid wall. The wide variety of practical interest applications, ranging from steam generation and condensation in power plants to process heating and products cooling in chemical industries, serves as motivation for the design optimization of these equipments (Fraas, 1989).

Heat exchangers are usually designed having a desired heat load and an allowable pressure drop across the device as the primary constraints (Kern, 1965). Therefore, a thermal and hydrodynamical analysis of the equipment becomes an important part of the design effort. Shell-and-tube heat exchangers are usually built from circular tubes, mounted in a predefined arrangement forming the tube bundle, and an involving cylindrical shell. Tube and shell axes are usually parallel. The knowledge of the geometrical characteristic parameters of the tube bundles, such as the tube count, which corresponds to the maximum number of tubes that can be accommodated within a specified shell, are important design data usually obtained from empirical correlations or tables (Saunders, 1988).

Applications of practical interest for which tables for the tube bundle geometrical parameters 
are not available or that fall outside the applicability range of the empirical correlations lead to the requirement of drafting the tube arrangement within the shell by hand or computer. Due to the increasing need for compact heat exchangers, situations where traditional design tools are not available are becoming common, leading to the development of new Engineering design tools. Besides, it is usually not possible to provide exact tube count tables due to the large number of dependent variables involved. Therefore, tube count tables found in the literature are usually applied only during the early design stages (Saunders, 1988).

A simple geometry-based computational procedure for the determination of tube bundle dimensional parameters is presented in the present work and applied to single and multiple tube-pass tubular heat exchangers. Initially, a searching procedure is used, allowing the precise determination of the possible tube center positions. Through the validation of the possible tube positions, the tube count for a given configuration is determined. A sorting procedure is used to appropriately place the tubes according to the tube distance to the shell center. The proposed computational procedure accounts for the effects of inlet and outlet nozzle diameters and relative nozzle position on the tube bundle distribution and for the presence of impingement plates. Tube bundle layout drawing is also provided for both the maximum and user-defined number of points. The parameters calculated by the proposed procedures can be used for thermal and mechanical design of heat exchangers and in performance analysis of operating equipment.

\section{TUBE POSITION SEARCH PROCEDURE}

A sketch of the tube searching procedure is depicted in Fig. 1, which also shows principal dimensions and the Cartesian system of coordinates used in the present work.

As for the hand drafting of the tube bundle layout, possible tube center positions are defined by the intersection of two straight lines which are used to scan the heat exchanger cross section by varying the straight line linear coefficients. The straight lines have angular coefficients of different signals. These angular coefficients are defined from the tube bundle and heat exchanger geometric parameters. In Fig. 1, the straight line with positive angular coefficient is used to scan the heat exchanger cross section from left to right while the negatively inclined searching line scans the cross section from right to left. The equations of the inclined straight lines used during the searching procedure are defined in general form as:

$$
y=a x+b ; a>0, l_{\max }>b>l_{\text {min }}
$$

and

$$
y=c x+d ; c<0, l_{\max }>d>l_{\min }
$$

and a possible tube position of order $k$ has coordinates defined by:

$$
\begin{aligned}
& x_{k}=\frac{d_{i}-b_{j}}{a-c} ; \\
& y_{k}=a\left(\frac{d_{i}-b_{j}}{a-c}\right)+b_{j}
\end{aligned}
$$

The tube-center to shell-center distance, which is used in the searching procedure, is defined by:

$$
\Delta_{k}=\sqrt{x_{k}^{2}+y_{k}^{2}}
$$

In order to completely scan the heat exchanger cross section, the limiting values of the straight line linear coefficients are defined as:

$$
l_{\max }=\frac{D_{S}}{2 \cdot \cos \theta}
$$

and

$$
l_{\min }=-\frac{D_{S}}{2 \cdot \cos \theta}
$$

where $D_{S}$ is the heat exchanger shell inside diameter.

For the tube searching procedure, the linear coefficient increment, which correspond to twice the distance between tube rows, is written as:

$$
\Delta l=2 \delta \sin \theta
$$

where $\delta$ is the tube pitch. The angular coefficients 


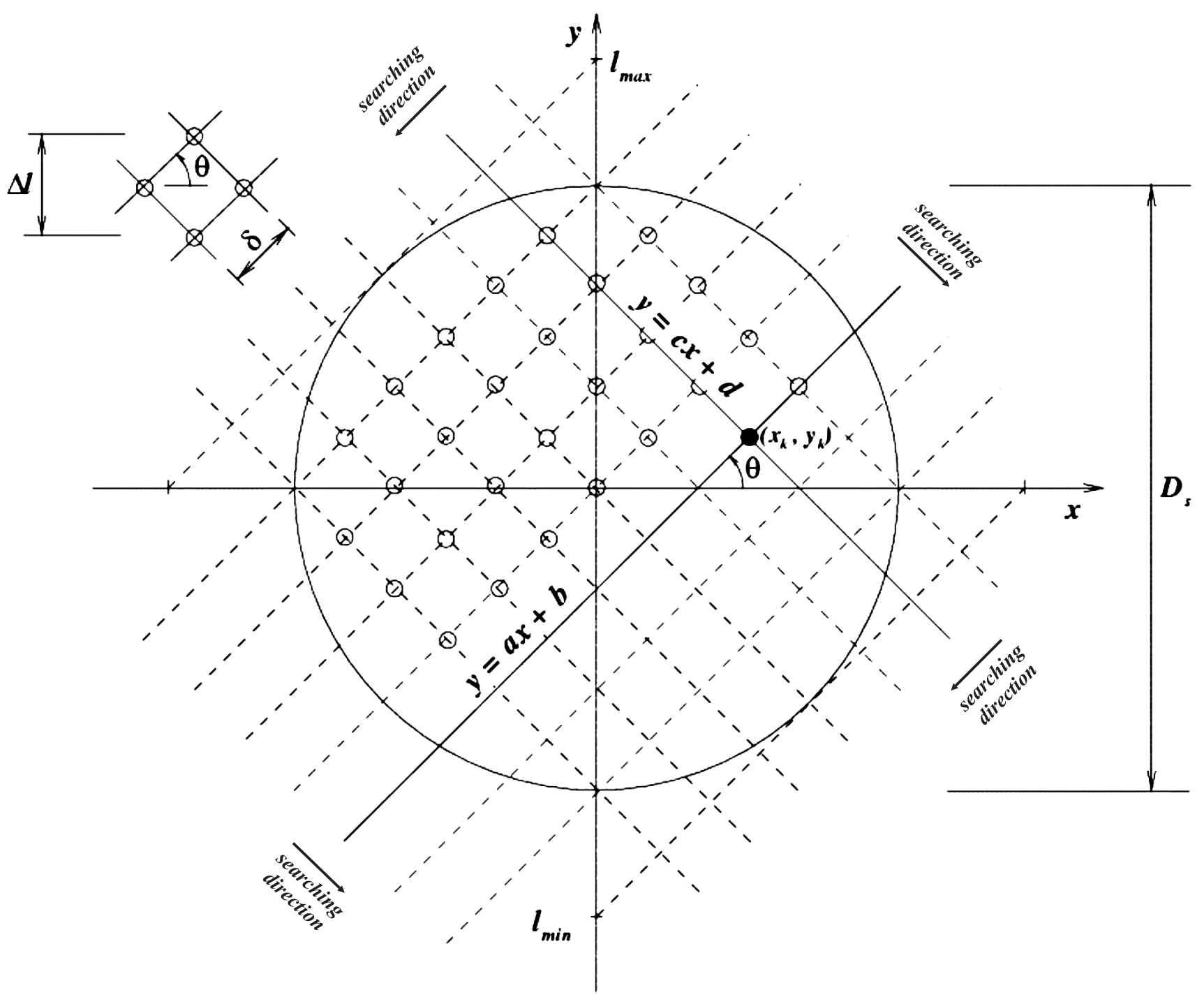

Figure 1. Sketch of the tube searching algorithm showing current (solid) and possible (dashed) searching line positions, previous $(\circ)$ and current $(\bullet)$ found tube positions and principle dimensions

of the searching lines $a$ and $c$ are defined as:

$$
a=\tan \theta
$$

and

$$
b=-\tan \theta
$$

It is worth mentioning that the angle $\theta$ is the complementary of the usually defined angle of the arrangement $\alpha$.

Once found by the geometrical procedure described above, a possible tube position must be validated in order to guarantee that it can accommodate a tube. At this stage of the algorithm, different criteria can be introduced, based on the heat exchanger configurations being addressed. In the present work, two restrictions are imposed on the initially found tube position. A radial condition for a valid tube position can be written as:

$$
\Delta_{k}+\frac{D_{t}}{2} \leq \frac{D_{S}}{2}-\varepsilon_{r}
$$

where $D_{t}$ is the tube outside diameter and $\varepsilon_{r}$ corresponds to the radial clearance between the tube bundle and the inner shell wall. The radial clearance may be defined by fouling constrains or the presence of sealing strips on the shell side.

Tube vibration and erosion also impose conditions on tube placement. A reduction on the number of tube rows that effectively can be defined for a heat exchanger configuration can be expected due to inlet and outlet nozzles and the use of impingement plates. The use of externally fitted impingement plates and distributors avoids the 
effective row number reduction, but those devices are not considered in the present work. The plate position is chosen in order to guarantee an annular escape area at least equal to the nozzle crosssection. Therefore, the position of the last row of tubes $\left(y_{\max }\right)$ is given as:

$$
y_{\max }=\frac{D_{S}}{2}-\frac{\phi_{i n}}{4}-t_{p}-\varepsilon_{t p}-\frac{D_{t}}{2}
$$

where $t_{p}$ is the impingement plate thickness and $\varepsilon_{t p}$ is the clearance between the impingement plate and the tube walls. The impingement plates are usually $6 \mathrm{~mm}$ thick with dimensions slightly wider than the inlet nozzle internal diameter $\left(\phi_{i n}\right)$. A fluid escape area must also be provided at the outlet nozzle and the position of the first row of tubes $\left(y_{\text {min }}\right)$ is given as:

$$
y_{\text {min }}=-\frac{D_{S}}{2}+\frac{\phi_{\text {out }}}{4}+\frac{D_{t}}{2}
$$

where $\phi_{\text {out }}$ is the outlet nozzle internal diameter, since impingement plates are usually not necessary at the shell outlet. A similar expression to Eq. 13 is applied for if the use of an impingement plate is unnecessary at the inlet nozzle. Therefore, a possible tube position will be validated if

$$
y_{\min } \leq y_{k} \leq y_{\max }
$$

Once finished, the searching procedure allows the determination of the heat exchanger tube count, tube bundle layout and the maximum outer tube limit for a given configuration. Nevertheless, further calculations are necessary if a given number of tubes is specified for the heat exchanger and the other tube bundle parameters are to be determined.

\section{TUBE DISTANCE SORT PROCEDURE}

The described tube searching procedure leads to a particular distribution of tube center distances. As shown in Fig. 2 for a 3410-tube heat exchanger, tubes located closer to the shell center are found halfway into the searching procedure. Tube positions with high are determined during the starting and finishing stages of the searching procedure.

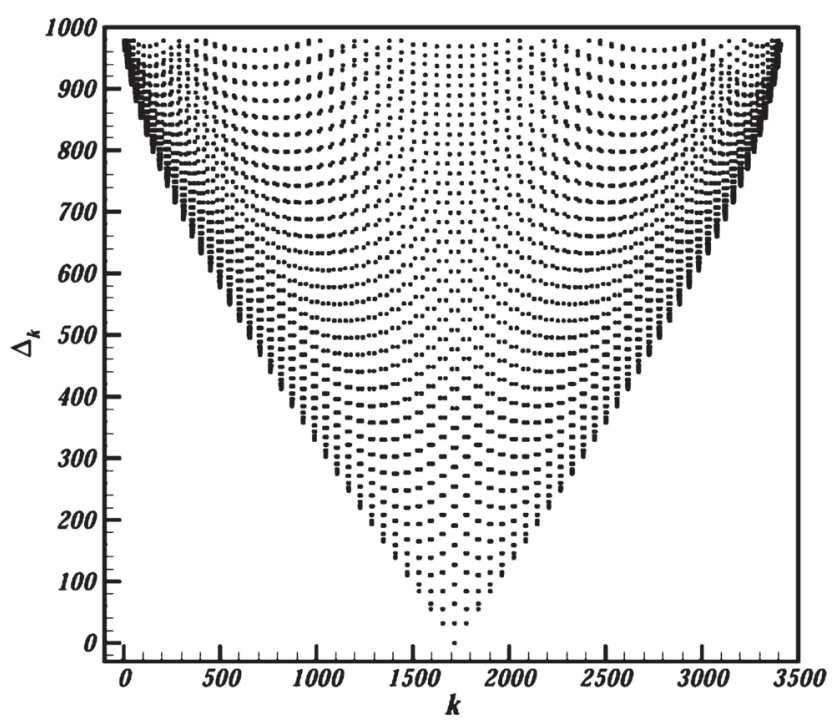

Figure 2. Typical $\Delta_{k}$ distribution after searching procedure $-D_{S}=2000 \mathrm{~mm}, D_{t}=25.4 \mathrm{~mm}, \alpha=60^{\circ}$ and $\phi_{\text {in }}=\phi_{\text {out }}=250 \mathrm{~mm}$, with impingement plate

If a given number of tubes is to be placed within the heat exchanger cross section, validated tube positions must be occupied in increasing order of tube center distances $\left(\Delta_{k}\right)$. Therefore, an efficient sort procedure must be used in order to adapt the initially found tube distribution to the given requirements.

In the present work, different sorting algorithms are applied to the $\Delta_{k}$ distribution obtained from the tube position search procedure. Initially a sequential sorting procedure, in which tubes of order $k$ and $k+1$ are switched if $\Delta_{k}>\Delta_{k+1}$, is used. However, the sequential or bubble sorting procedure can become computationally inefficient (Knuth, 1998), especially for heat exchangers with large number of tubes. In order to provide a basis for comparison between different sorting methods, a 39,697-tube heat exchanger is used as reference. The sorting procedure for the reference heat exchanger using the sequential sorting algorithm took more than $60 \mathrm{~s}$ before being completed on an AMD $1.3 \mathrm{GHz}$ computer.

Instead of neighboring elements, the Shell's sorting method compares elements that are located apart in the unsorted data set (Knuth, 1998). For the current work, the distance between elements being compared is initially half the maximum number of tubes. The offset value is continuously 
halved after each data sweep, until a unitary offset value is reached. At this point a bubble sort sweep is performed. For the reference heat exchanger with 39,697 tubes, data sorting by the Shell's method is completed in $2.0 \mathrm{~s}$, on the same AMD $1.3 \mathrm{GHz}$ computer.

The Quicksort algorithm (Knuth, 1998), for which pointers are assigned to two tube positions, is also tested. For the present work, the first and last found tube positions are used as initial pointers. The sorting procedure consists of comparing $\Delta_{k}$ for the pointers and switching the tube order appropriately. If the comparison of the tube distances to center leads to a no-switching situation, one of the pointers is moved towards the center of the distribution. The moving pointer is alternated after each tube order switch. The Quicksort algorithm leads the first found tube to be placed in the correct position within the final distribution after the first distribution sweep. Besides, the Quicksort algorithm also splits the tube distribution based on $\Delta_{k}$ after each sweep. Quicksort is then applied to each individual data set (Knuth, 1998). For the reference heat exchanger and hardware, Quicksort required $1 \mathrm{~s}$ to complete sorting the original data set.

\section{PROCEDURE FOR MULTIPLE TUBE-PASS HEAT EXCHANGERS}

The tube position searching procedure for single and multiple pass heat exchangers are similar. The difference resides in considering additional constrains introduced by pass partition plates with finite thickness. The pass partition constrains are considered during the tube position validation step.

In order to explain the extension of the proposed procedure for multiples pass heat exchanger, a two-pass heat exchanger is initially considered. For the given configuration, a pass partition is positioned along the heat exchanger longitudinal axis, splitting the cross section into two equal halves. Tube positions obtained assuming single pass are revalidated based on the vertical position. A tube vertical position $\left(y_{k}\right)$ is considered valid if

$$
y_{k}<y_{\max } \text { and } y_{k}>t_{c}+\frac{D_{t}}{2}
$$

for the upper half and

$$
y_{k}>y_{\min } \text { and } y_{k}<-\left(t_{c}+\frac{D_{t}}{2}\right)
$$

for the lower half, where $t_{c}$ represents the thickness of the pass partition plate. Finally, the number of tubes in each pass is compared. If the exchanger configuration leads to discrepancies in the tube count per pass, the smallest of the calculated number of tubes is used.

The equalization of the number of tubes per pass is also used as a criterion for positioning the pass partition plate for heat exchangers with more than two tube-passes. Initially, the heat exchanger section is split into subsections of equal height. The number of subsections is equal to the tube-passes prescribed for the heat exchanger. From the position of the subsection interfaces and the pass partition plate thickness, constrains similar to the one in Eqs. (15)-(16) can be defined and applied to the tube distribution established for a single-pass heat exchanger with the same configuration. The tube count for each pass is determined and compared. Discrepancies are corrected by repositioning the pass partition plates accordingly and recalculating the tube distribution. The iterative process ends when the difference between the pass tube-counts is smaller than a user-defined tolerance or a maximum number of iterations is reached.

\section{RESULTS}

The implementation of the searching and sorting procedures was performed using the Delphi 3 Integrated Development Environment (IDE) (Cantú, 1997), object oriented programming and a simple and practical Human Computer Interface (HCI) (Pressman, 1995). The choice of the IDE made available the Unit Math, which contains subroutines specially compiled for the Floating Point Unit (FPU) of Pentium processors (Taylor, 1999). Besides, the Delphi 3 IDE is known to have appropriate interacting performance with the Graphics Display Interface (GDI) of the Microsoft Windows Operating System.

Results for different arrangement angles are shown in Figs. 3 and 4 for a base configuration of

$$
\begin{aligned}
& D_{s}=635 \mathrm{~mm}, \quad D_{t}=25.4 \mathrm{~mm}, \quad \delta=31.75 \mathrm{~mm}, \\
& \varepsilon_{r}=3.175 \mathrm{~mm} \text { and } \phi_{\text {in }}=\phi_{\text {out }}=203 \mathrm{~mm} \quad \text { with an }
\end{aligned}
$$


impingement plate placed along the inlet nozzle. For the given configuration, results show that 296, 270,292 and 268 tubes can be used for $\alpha=30^{\circ}$, $45^{\circ}, 60^{\circ}$ and $90^{\circ}$, respectively. Tube count tables for $\alpha=30^{\circ}$ (Saunders, 1988) indicate, for specified values of $D_{s}, D_{t}$ and $\delta$, a maximum of 311 tubes, corresponding to a $4.8 \%$ deviation from the calculated value. It is noteworthy the influence of radial clearance $\varepsilon_{r}$ on the calculated results. As

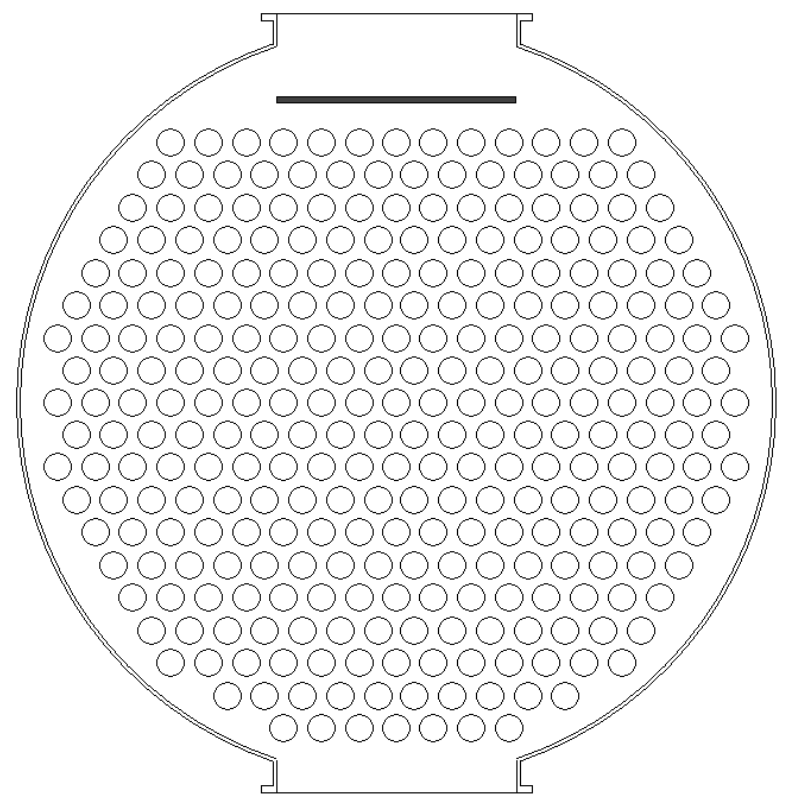

(a) $\varepsilon_{r} \rightarrow 0$, the difference between calculated and tube count table values tends to vanish. Nevertheless, the value of $\varepsilon_{r}$ used by Saunders (1988) in developing the data tables is unclear. The use of a $30^{\circ}$ layout usually leads to the highest tube density and heat transfer intensity for a given configuration and becomes the primary layout choice during heat exchangers design despite the associated pressure loss.

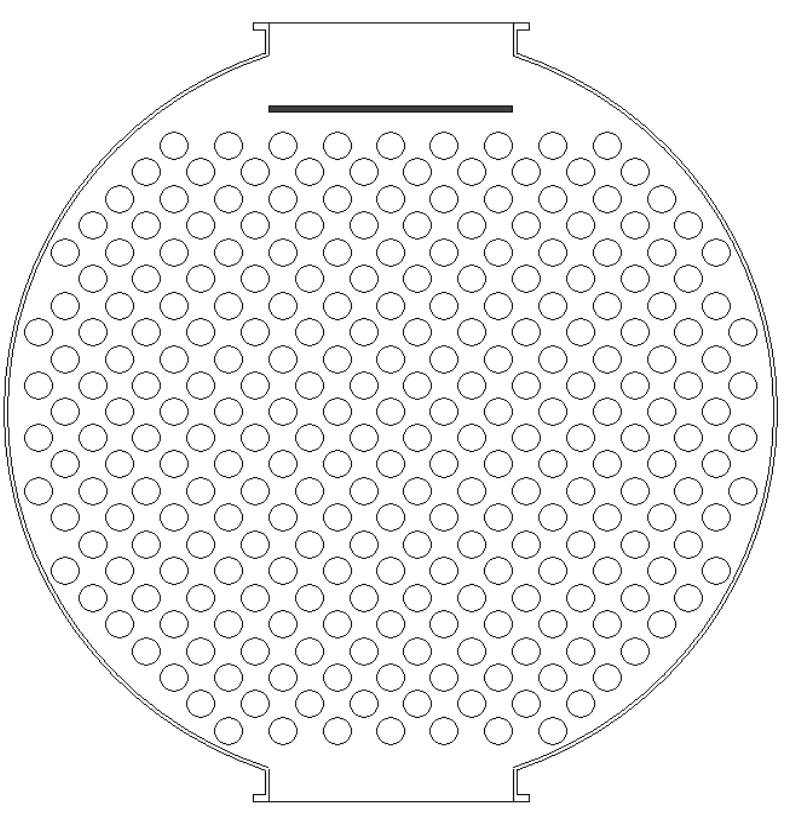

(b)

Figure 3. Tube bundles for $D_{s}=635 \mathrm{~mm}, D_{t}=25.4 \mathrm{~mm}, \delta=31.75 \mathrm{~mm}, \phi_{\text {in }}=\phi_{\text {out }}=203 \mathrm{~mm}$ and $\varepsilon_{r}=3.175 \mathrm{~mm}$ with $\alpha=30^{\circ}$ (a) and $45^{\circ}$ (b)

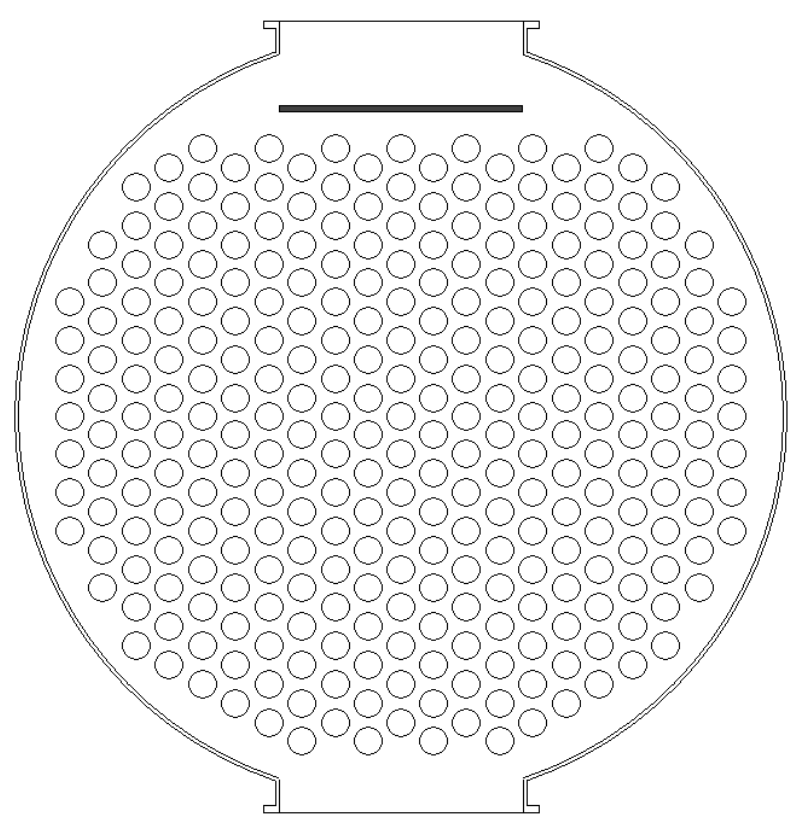

(a)

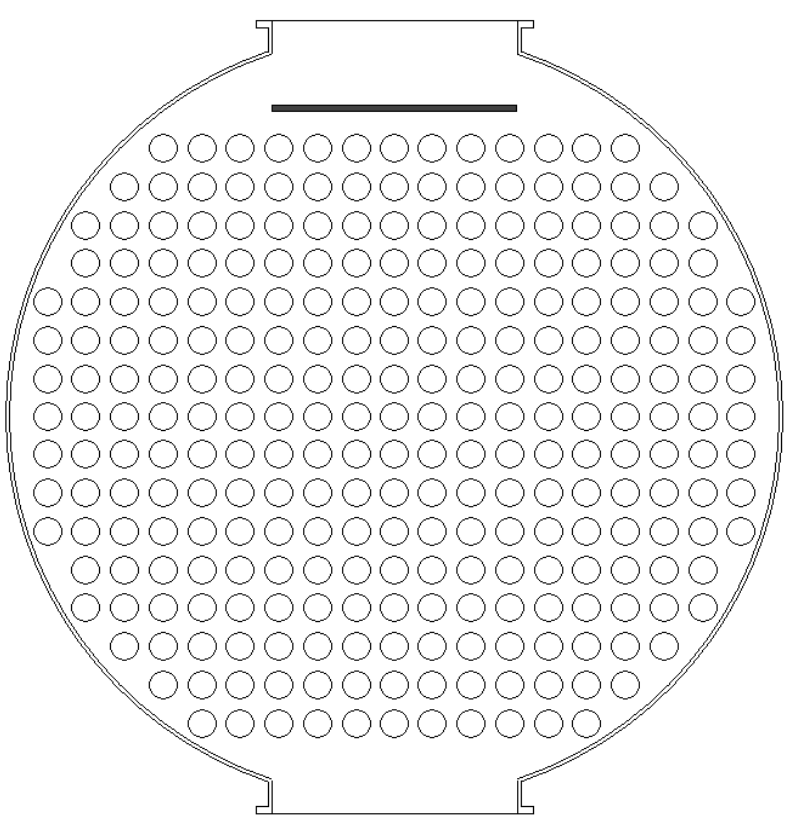

(b)

Figure 4. Tube bundles for $D_{s}=635 \mathrm{~mm}, D_{t}=25.4 \mathrm{~mm}, \delta=31.75 \mathrm{~mm}, \phi_{\text {in }}=\phi_{\text {out }}=203 \mathrm{~mm}$, and $\varepsilon_{r}=3.174 \mathrm{~mm}$ with $\alpha=60^{\circ}$ (a) and $\alpha=90^{\circ}$ 


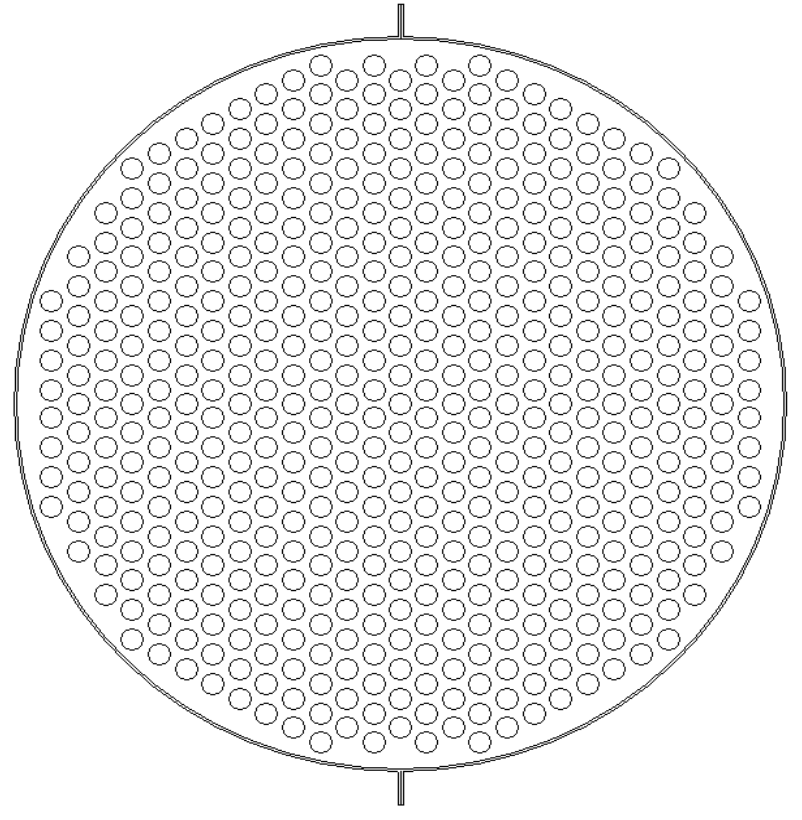

(a)

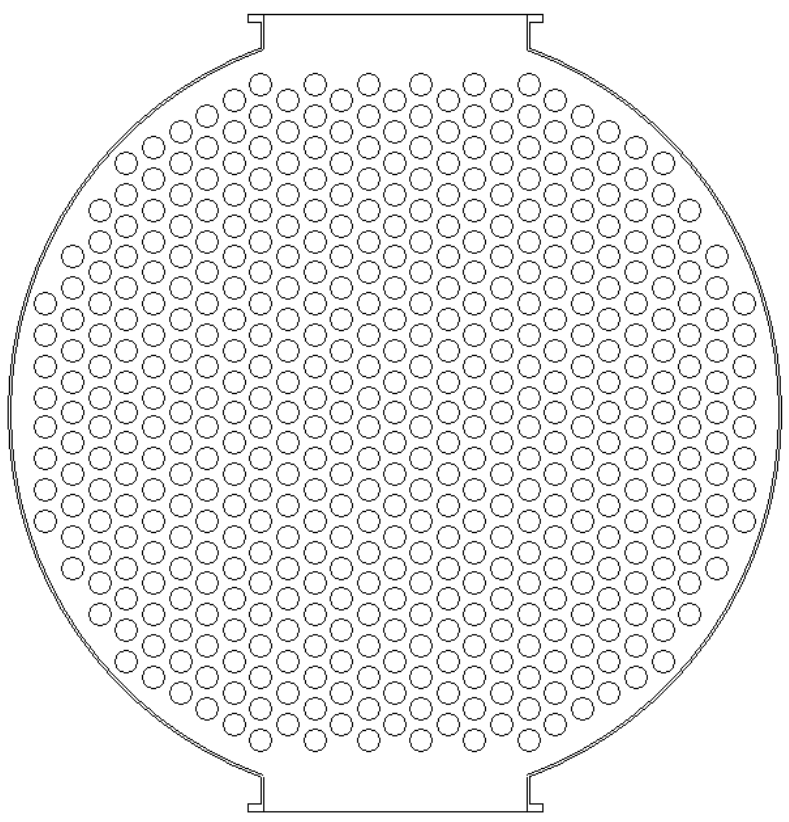

(b)

Figure 5. Tube bundles for $D_{S}=591 \mathrm{~mm}, D_{t}=19.05 \mathrm{~mm}, \delta=23.81 \mathrm{~mm}, \alpha=60^{\circ}$, $\varepsilon_{r}=3.175 \mathrm{~mm}-\phi_{\text {in }}=\phi_{\text {out }}=0.0 \mathrm{~mm}$ (a) and $\phi_{\text {in }}=\phi_{\text {out }}=203 \mathrm{~mm}$ (b)

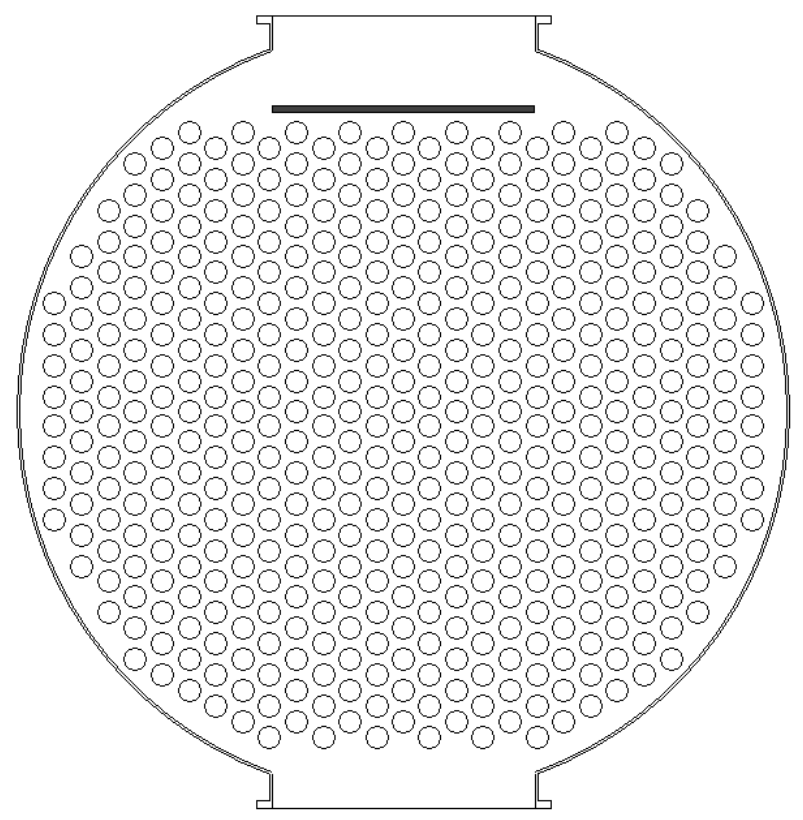

(a)

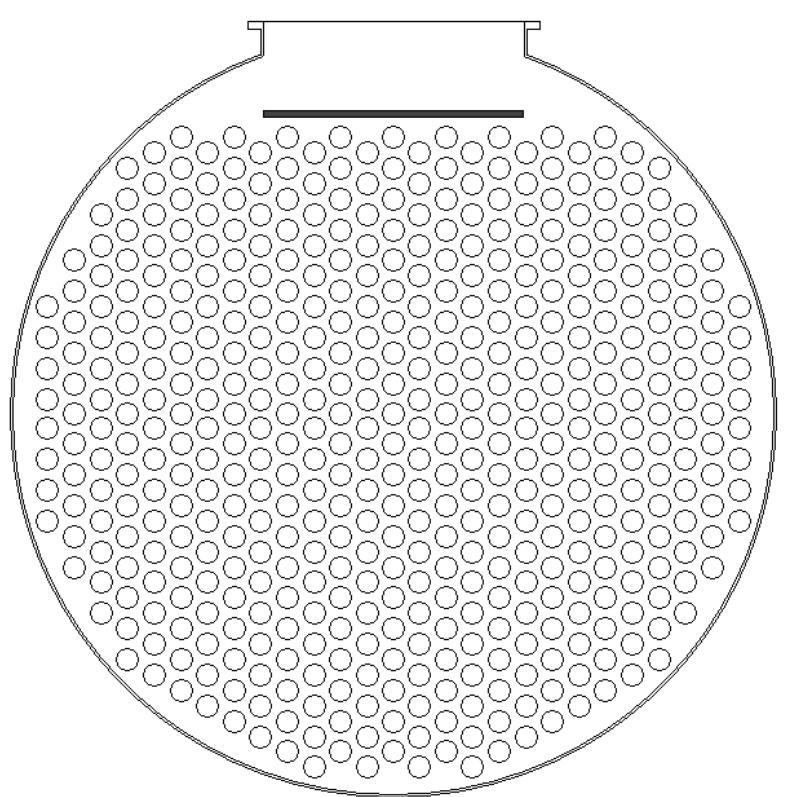

(b)

Figure 6. Tube bundle for $D_{S}=591 \mathrm{~mm}, D_{t}=19.05 \mathrm{~mm}, \delta=23.81 \mathrm{~mm}, \alpha=60^{\circ}, \varepsilon_{r}=3.175 \mathrm{~mm}$, impingement plate - Inlet and outlet nozzles at different sides (a) and at the same side of heat exchanger shell (b)

In order to further analyze the influence of different parameters involved, results of the implemented algorithm are also shown in Figs. 57 for a second base configuration of $D_{s}=591 \mathrm{~mm}$, $D_{t}=19.05 \mathrm{~mm}, \quad \delta=23.81 \mathrm{~mm}, \quad \alpha=60^{\circ} \quad$ and $\varepsilon_{r}=3,175 \mathrm{~mm}$. Considering $\phi_{\text {in }}=\phi_{\text {out }}=0.0 \mathrm{~mm}$, a tube count of 511 is obtained, while data tables (Saunders, 1988) indicate that a maximum of 516 tubes ( $1 \%$ deviation) can be accommodated within the heat exchanger shell. A sketch of the tube bundle layout is shown in Fig. 5(a) for the 511tube configuration. Considering inlet and outlet nozzle diameters equal to $203 \mathrm{~mm}$, calculations show that 493 tubes can be accommodated and the corresponding tube layout is shown in Fig. 5(b). If an impingement plate is placed at the inlet nozzle position and $\phi_{\text {in }}=\phi_{\text {out }}=203 \mathrm{~mm}$, the maximum 


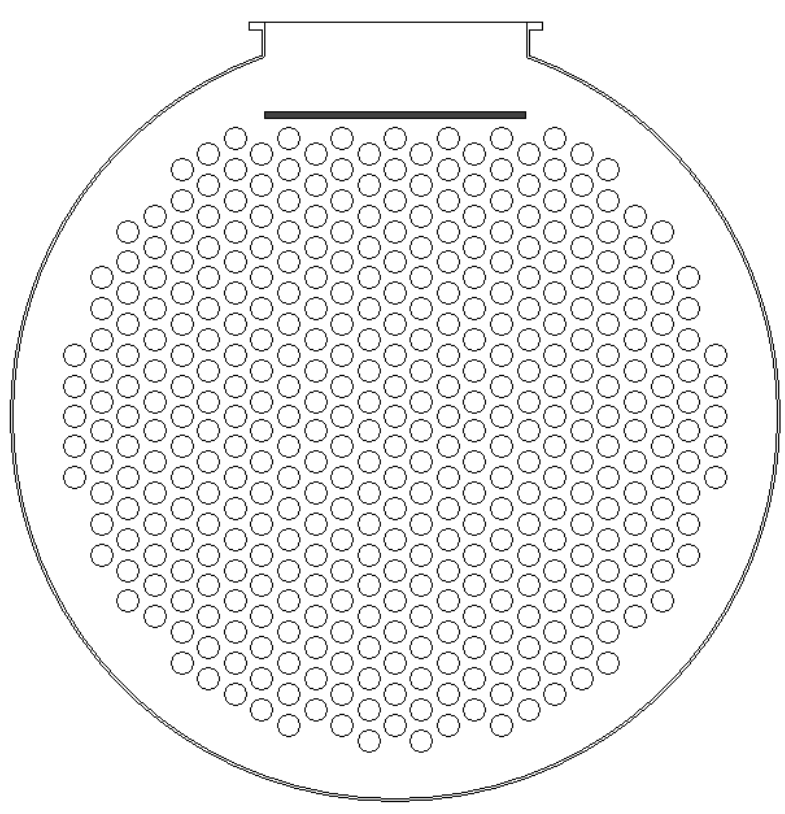

(a)

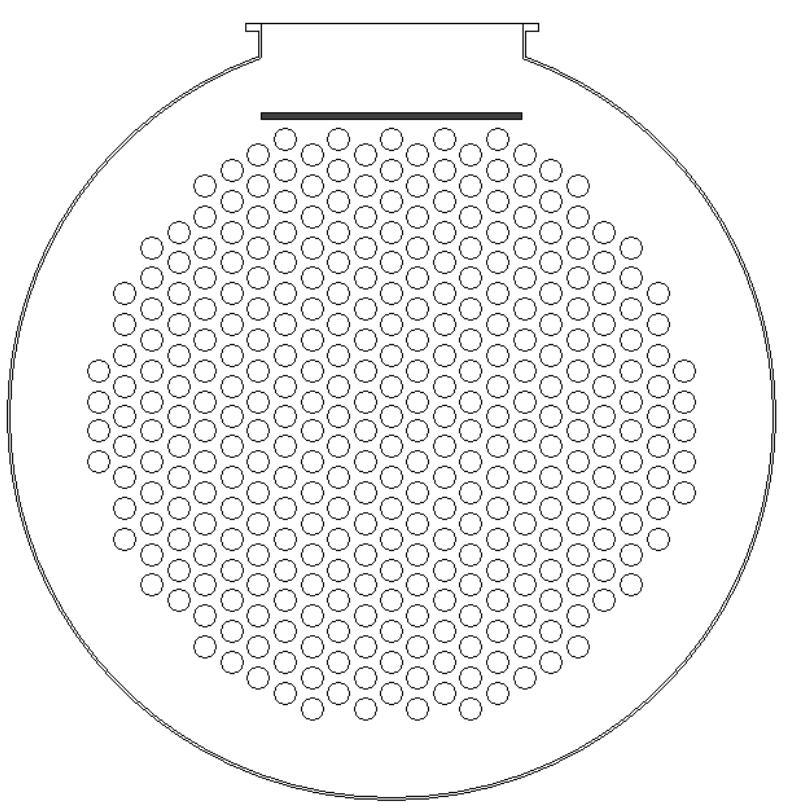

(b)

Figure 7. Tube bundles for $D_{s}=591 \mathrm{~mm}, D_{t}=19,05 \mathrm{~mm}, \delta=23,81 \mathrm{~mm}, \alpha=60^{\circ}, \varepsilon_{r}=3,175 \mathrm{~mm}$ and $\phi_{\text {in }}=\phi_{\text {out }}=203 \mathrm{~mm}-408$ tubes (a) and 350 tubes (b)

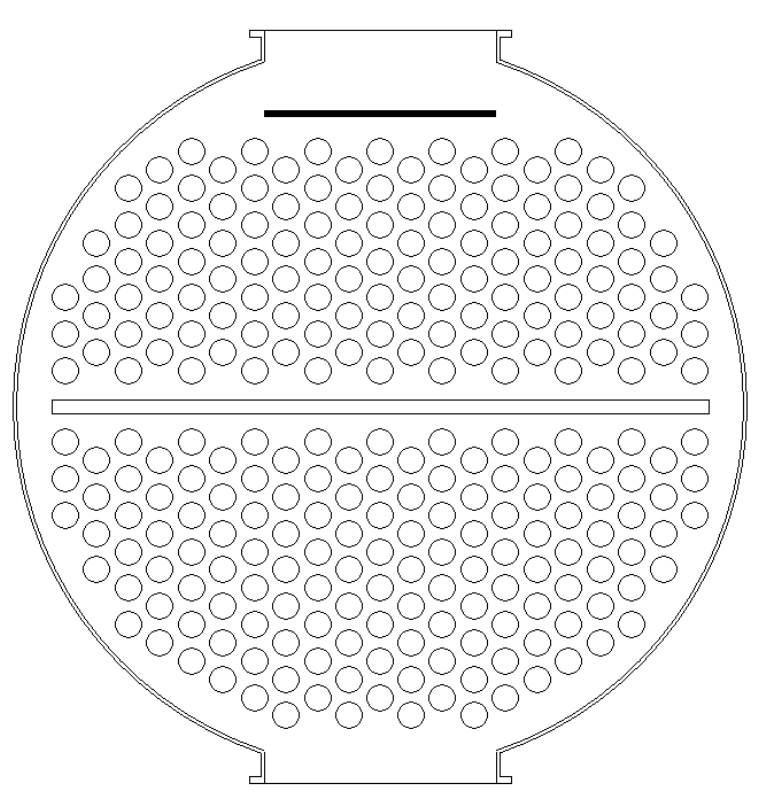

(a)

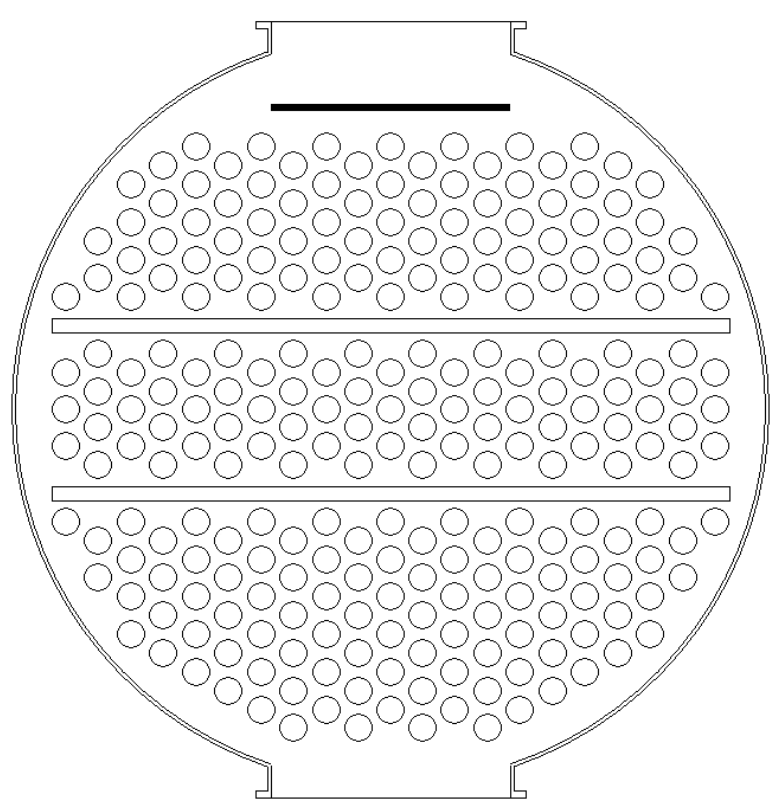

(b)

Figures 8. Tube bundles for $D_{s}=635 \mathrm{~mm}, D_{t}=25.4 \mathrm{~mm}, \delta=31.75 \mathrm{~mm}, \varepsilon_{r}=3.175 \mathrm{~mm}, \phi_{\text {in }}=\phi_{\text {out }}=203 \mathrm{~mm}$ and $\alpha=60^{\circ}$, considering (a) one and (b) two longitudinal dividing plates

number of tubes is reduced to 472 , as shown in Fig. 6(a). Placing the inlet and outlet nozzle at the same side of the heat exchanger shell, as depicted in Fig. 6(b), allows the addition of 9 tubes to the previous bundle.

For the second base configuration, cases where the number of tubes to be accommodated is smaller than the calculated maximum shell capacity of 472 tubes are illustrated in Fig 7(a), where 408 tubes are used, leading to a calculated outer tube limit (OTL) of $534.2 \mathrm{~mm}$. The outer tube limit corresponds to the diameter of the largest circle drawn around the tubesheet center, which is tangent to the most externally placed tube. Reducing the number of tubes to 350 , the outer tube limit is reduced to $448.0 \mathrm{~mm}$, and a sketch of the tube bundle layout is depicted in Fig 7(b).

Results for heat exchangers with the second base configuration and longitudinal dividing plates are depicted in Fig. 8. Dividing plates with $6 \mathrm{~mm}$ 


\section{TECNOLOGIA/TECHNOLOGY}

thickness are considered and the number of tubes per pass equalization procedure is relaxed. In tube bundle shown in Fig. 8(a), 123 and 138 tubes are accommodated in each tube pass. The lower tube count is associated with the inlet nozzle impingement plate. Tube count tables (Saunders, 1988) indicate that 298 tubes can be accommodated, leading to a $12 \%$ discrepancy with the calculated results. Reducing the tube radial clearance $\left(\varepsilon_{r}\right)$ to zero, the results from the proposed procedure indicate a tube count of 273 tubes $(8.4 \%$ discrepancy). The discrepancy between the obtained and data table tube counts is reduced to $0.33 \%$ if the partition plate thickness is reduced to $3 \mathrm{~mm}$. In Fig. 8(b) a non-conventional heat exchanger configuration with two longitudinal dividing plates is shown. For this configuration, the results show that 81,76 and 96 tubes can be placed near the shell inlet nozzle, at the center part of the heat exchanger and near the shell outlet nozzle, respectively. In contrast with the results obtained in Fig. 8(a), varying the dividing plate thickness has no influence on the obtained results.

\section{CONCLUSIONS}

A computational procedure for the determination of tube bundle parameters for tubular heat exchangers is introduced and tested. Initially, a tube position searching algorithm is discussed and the validation criteria for the found positions defined for a given equipment configuration. A sorting procedure based on the tube distance to the shell center is also introduced in order to address design situations where the required number of tubes is smaller than the heat exchanger tube count. Due to the distribution of tube distances obtained from the searching procedure, sorting algorithms that compare tubes located far apart within the original distribution require less computational times. Nevertheless, the difference in computing time due to the sorting procedure becomes important only for heat exchangers with large number of tubes. The extension of the discussed methodology to multiple tube pass heat exchangers is also discussed based on horizontal pass partition plates. Results for one- and two-tube pass heat exchangers are presented showing features of the implemented procedure and the precision of the calculations, when compared to tube count table data. Tube counts discrepancies of $1 \%$ were observed between calculated and table values. Further developments are required in order to extend the procedure to more general situations by incorporating features commonly found in industrial heat exchangers and to allow for thermohydraulic analysis of the obtained results (Taborek, 1983). Computational times are also measured for the obtained tube bundles and the implemented sorting subroutines. It was observed that computational times differences associated with the bubble-sort and Quicksort algorithms become smaller than the precision of the subroutine employed for the measurement.

\section{ACKNOWLEDGEMENTS}

The authors wish to acknowledge the financial support provided by CNPq.

\section{REFERENCES}

Cantú, M., 1997, Mastering Delphi 3, Makron Books, São Paulo.

Fraas, A. P., 1989, Heat Exchanger Design, John Wiley, New York.

Kern, D. Q., 1965, Process Heat Transfer, McGraw Hill, New York.

Knuth, D. E., 1998, The Art of Computer Programming: Sort and Searching, Vol. 3, Addilson-Wesley, New York.

Pressman, R., 1995, Engenharia de Software, Makron Books, São Paulo. (in Portuguese)

Saunders, E. A. D., 1988, Heat Exchangers Selection, Design and Construction, Longman Scientific \& Technical, Harlow, Essex, England.

Taborek, J., 1983, Heat Exchanger Design Handbook, Hemisphere, New York.

Taylor, D., 1999, Programando Delphi 3 Técnicas Avançadas, Makron Books, São Paulo. (in Portuguese) 